\title{
Article
}

\section{Intercalation Compounds of Layered Titanium Phosphate for Drug Delivery Use}

\author{
Tomoko TAJIMA, Noriko SUZUKI, Yoshiteru WATANABE and Yasushi KANZAKI \\ Showa Pharmaceutical University, 3-3165 Higashi-Tamagawagakuen, Machida, Tokyo 194-8543, Japan
}

(Manuscript submitted Feburary 15, 2003; accepted May 6, 2003)

\begin{abstract}
Abstruct
Layered inorganic material, $\gamma$-titanium phosphate $(\gamma$-TiP), was applied as a host material for drug delivery use. Hygroscopic diclofenac sodium (DFS) was selected as a model drug to improve its anti-humidity and anti-oxidation. The intercalation was carried out in ethanol solution of DFS. Propylamine was used as the third compound to expand interlayer gallery and/or exfoliate lamellae. The amount of intercalated DFS was almost independent on the concentration of propylamine added. The drug release characteristics was also investigated. The release rate of DFS from the intercalation compound was slower than DFS powder and almost all DFS intercalated was released within $120 \mathrm{~min}$.
\end{abstract}

\section{Introduction}

Some layered inorganic compounds can accommodate polar organic compounds between the layered lamellae and form a variety of intercalation compounds. The host compounds must be harmless to the human body when the intercalation compounds were applied to medical use. One of the promising inorganic materials as such host compound is layered $\gamma$-titanium phosphate, $\mathrm{Ti}\left(\mathrm{HPO}_{4}\right)_{2} \cdot 2 \mathrm{H}_{2} \mathrm{O}(\gamma$-TiP). Figure 1 shows SEM photograph of crystalline titanium phosphate prepared hydrothermally. $\gamma$ TiP is known to exfoliate by the topo chemical reaction in the presence of $\mathrm{n}$-propylamine similar to $\alpha$-zirconium phosphate.[1] The exfoliated $\gamma$-TiP lamellae is reassembled again to remove solvent (Fig.2). The intercalation of molecules with a large molecular weight to $\gamma$-TiP can occur during the exfoliation-reassemble process. In this study, we have focused on a hygroscopic drug as a guest compound to verify the anti-humidity and antioxidation of the molecules accommodated in the interlayer space. The drug was diclofenac sodium abbreviated to DFS; sodium $[o-[(2,6-$ dichlorophenly)amino]phenyl]acetate. DFS is widely used as an antiinflammatory agent, especially for the rheumatism.

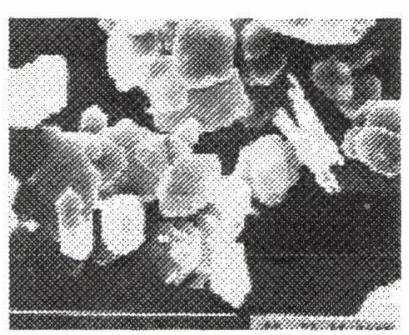

Fig. 1 SEM photograph of titanium phosphate

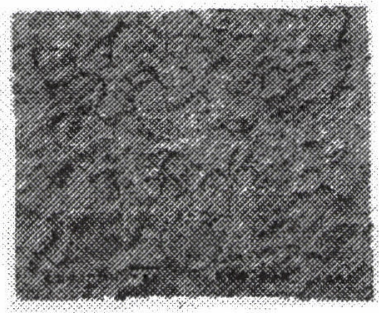

Fig. 2 SEM photograph of exfoliated titanium phosphate 


\section{Experimental}

\subsection{Precursor}

Amorphous titanium phosphate was prepared in advance as reported.[2] Crystalline dihydrated titanium(IV) phosphate was obtained by hydrothermal synthesis as follows.[3] $1.0 \mathrm{~g}$ of amorphous titanium phosphate and $20 \mathrm{~cm}^{3}$ of $10 \mathrm{~mol} \mathrm{dm}^{-3} \mathrm{H}_{3} \mathrm{PO}_{4}$ were placed in a glass tube and sealed. The tube was then put in an autoclave. Hydrothermal synthesis was carried out at $270^{\circ} \mathrm{C}$ and $7 \times 10^{6} \mathrm{~Pa}$ for $36 \mathrm{~h}$.

\subsection{Model drug}

DFS (m.p. $283-285^{\circ} \mathrm{C}$, UV max: $276 \mathrm{~nm}$ in phosphate buffer, purchased from Wako Pure Chemical Ind., Ltd.) was selected as a model drug and was used without further purification.

\subsection{Intercalation}

Intercalation was carried out in two processes to utilize the exfoliating phenomenon of the lamellae of the host $\gamma$-TiP layer.

Method 1: $\quad 2 \sim 35 \mathrm{~mm}^{3}$ of n-propylamine was added into suspended solution of $\gamma$-TiP $\left(0.1 \mathrm{~g} \gamma\right.$-TiP in $10 \mathrm{~cm}^{3}$ ultra purified water). Then $5 \mathrm{~cm}^{3}$ of saturated ethanol solution of DFS $\left(3.6 \times 10^{-2} \mathrm{~mol} \mathrm{dm}^{-3}\right)$ was added in the suspension. According to the mixing, a suspension of $\gamma$-TiP appeared to indicate the formation of exfoliated lamellae. The reaction time was $24 \mathrm{~h}$ and gel-like precipitate was formed. The precipitate was served for further measurement

Method 2: $0.058 \mathrm{~g}$ of DFS and a known amount of amine were dissolved in $10 \mathrm{~cm}^{3}$ ethanol in advance. Then $0.1 \mathrm{~g}$ of $\gamma$-TiP powder was added in the ethanol solution containing DFS. N-propylamine, $\mathrm{n}$-amylamine, $\mathrm{n}$ heptylamine $n$-decylamine and $n$-tetradecylamine were used. The reaction time was $24 \mathrm{~h}$ and the precipitate was served for further measurement.

In both processes, an aliquot of the supernatant solution containing residual DFS was taken out at a fixed period of time and the amount of DFS was measured by a spectrophotometer to calculate the amount of intercalated DFS.

For comparison, DFS intercalation was examined on synthetic mica (TSM)-phosphatidylcholine (PC) intercalation compound as a precursor in which intercalation of indomethacin was successfully occurred.[4] In this case, two procedures were also examined. In one procedure, TSM-PC intercalation compound was prepared in ethanol solution of PC. It had an interlayer gallery height of about $5 \mathrm{~nm}$. Saturated ethanol solution of DFS was then added in the solution to prepare TSM-PC-DFS intercalation compounds. In another procedure, ethanol solution of DFS-PC mixture was prepared in advance and Mica powder was added in the mixed solution. Cointercalation of DFS was expected on the occasion of PC intercalation.

\subsection{Characterization}

The products were characterized by using of a powder X-ray diffractometer (MO3X-HF, MAC Science Co., Ltd., Japan) equipped with monochromized $\mathrm{Cu}-\mathrm{K} \alpha$.

\subsection{Drug Release Property}

Drug release rate was monitored based on the Japanese Pharmacopoeia Thirteenth Edition paddle method. The pHs of the buffer solution were fixed at 3.0, 5.0, 7.0 and 10.0. The volume of the buffer solution was $500 \mathrm{~cm}^{3}$ and $0.032 \mathrm{~g}$ of drug or the intercalation compounds in which equivalent amount of DFS were contained. $3 \mathrm{~cm}^{3}$ of the test solution was sampled at fixed intervals and the amount of released drug was monitored using a spectrophotometer (UV-1600PC, Shimadzu Co., Japan). 


\section{Results and Discussion}

\subsection{XRD patterns of products}

Figure 3 shows the powder Xray patterns of products prepared. The interlayer distance of $\gamma$-TiP expanded to $1.61 \mathrm{~nm}$ by the intercalation of $\mathrm{n}$ propylamine (b). $\quad \gamma$-TiP + n-propylamine + DFS intercalation compound prepared by Method 1 has the diffraction peaks at $2 \theta=4.6$ and 6.8 with the interlayer distances of $1.90 \mathrm{~nm}$ and $1.29 \mathrm{~nm}$, respectively. The intercalation compound of $\gamma$-TiP and DFS ethanol solution had the diffraction peak at $2 \theta=$ 6.8 (data is not shown). From this fact, the interlayer distance of $1.29 \mathrm{~nm}$ was assigned to a $\gamma$-TiP/DFS compound without amine. It is confirmed that the

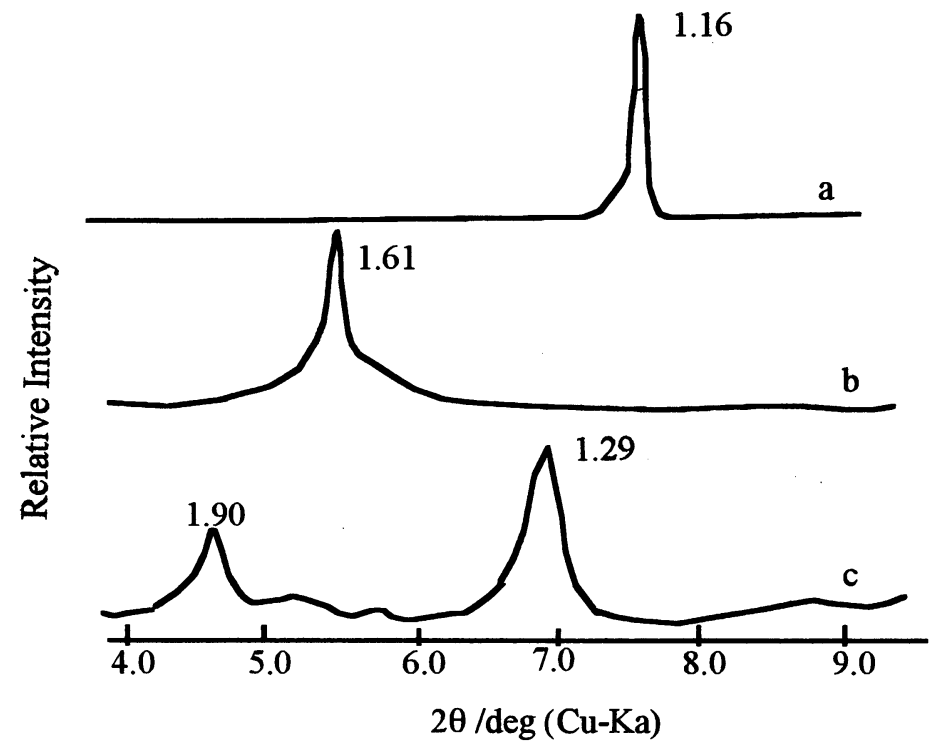

Fig. 3 Powder X-ray diffraction patterns. a: $\gamma$-TiP, b: $\gamma$-TiP + n-propylamine, c: $\gamma$-TiP + n-propylamine + DFS. amount of intercalated DFS did not participate in n-propylamine discussed below.

\subsection{Amount of intercalated DFS}

Method 2 was examined first for various nalkyl amines. However, no decrease in absorbance of DFS was observed in the supernatant solution. The fact meant no intercalation of DFS into $\gamma$-TiP occurred by Method 2. As a consequence, Method 1 was used in the rest of the present study.

To elucidate the superiority of the present intercalation procedures, DFS intercalation was examined on TSM in advance. Figure 4 shows the amount of DFS intercalated in TSM-PC intercalation compounds. The reaction was very slow and two days were necessary to reach equilibrium. The amount of DFS was very low comparing with indomethacin intercalation, about a few $\mathrm{mg}$ in $0.1 \mathrm{~g}$ of Mica.

The result obtained by Method 1 is shown in Fig. 5 in which the amount of intercalated DFS was examined at various concentration of $n$-propylamine. The intercalation of DFS reached equilibrium with in 12 $h$ for all cases. The amount of intercalated DFS in $\gamma$ TiP was about fourteen times larger than that in TSM shown in Fig. 4. No significant difference was observed up to $10 \mathrm{~mm}^{3}$ of n-propylamine added. The

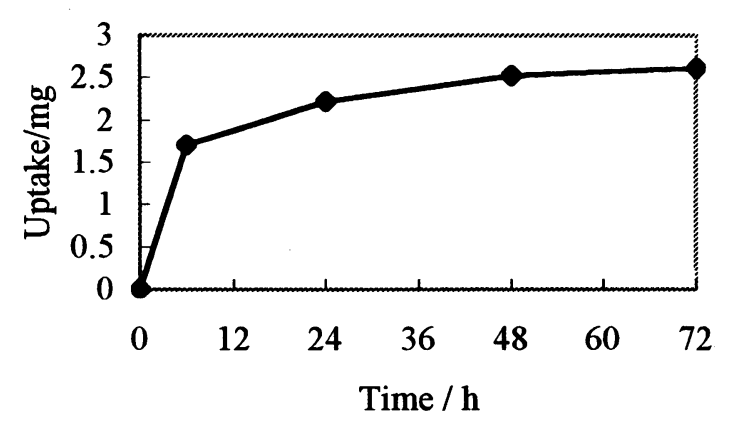

Fig. 4 Uptake of DFS to TSM-PC

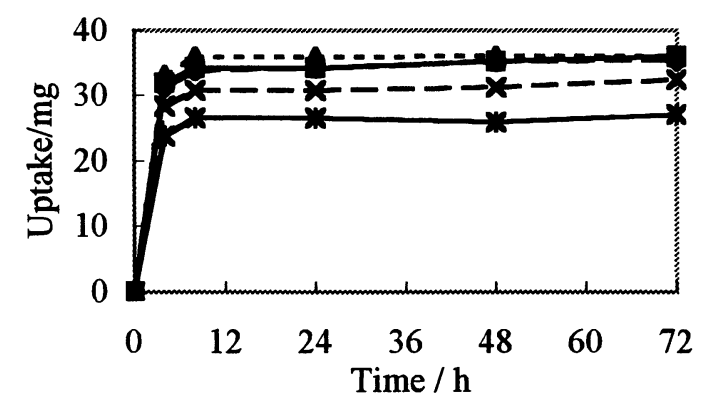

Fig. 5 Uptake of DFS to $\gamma$-TiP with various concentoration of $\mathbf{n}$-propylamine

Added amounot of $\mathrm{n}$-propylamine, given in $\mathrm{mm}^{3}$

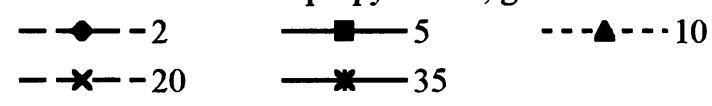


amount of DFS intercalated decreased a little with increasing the volume of $n$-propylamine when $n$-propylamine added exceeded $20 \mathrm{~mm}^{3}$. DFS was found to intercalate even in the absence of $n$-propylamine. This result indicated that intercalation of DFS was assisted mainly by coexisting ethanol. The effect of propylamine and other $\mathrm{n}$-alkyl amines was low contrary to our expectation. The phenomenon is completely different from the DFS intercalation on TSM (Fig. 4) and also indomethacin on TSM.[4]

\subsection{Drug Release Property}

The anti-humidity and anti-oxidation ability of DFS intercalated in TSM has already been proved.[5] Accordingly, the release property into solution phase was examined to elucidate the potentiality of $\gamma$-TiP-DFS intercalation compound as a drug delivery system. The release property from DFS powder and DFS intercalation compounds in neutral solution is shown in Fig. 6. DFS released from DFS powder into the buffer solution was fast and reach equilibrium within $5 \mathrm{~min}$. On the other hand, the release rate from $\gamma$ TiP-DFS intercalation compound was rather slow and over $40 \mathrm{~min}$ was necessary to reach a steady state. Similar property was observed in the buffer solution at $\mathrm{pH}$ 10.0. On the other hand, DFS was hardly

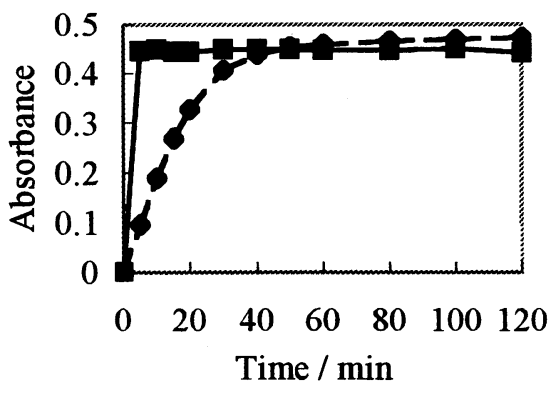

Fig. 6 Release property at $\mathrm{pH} 7.0$

$-\sim-$ Intercalation compound

$\longrightarrow$ DFS powder released from neither DFS powder nor $\gamma$-TiP-DFS intercalation compound in the acidic buffer solution at $\mathrm{pH}$ 3.0.

It was found from above results, $\gamma$-TiP-DFS intercalation compound prepared by Method 1 seems superior method for drug delivery use because $\gamma$-TiP used were all harmless to the human body in addition to its simplicity and high accommodated amount. In addition, release rate was also suppressed slow in comparison with DFS powder. Furthermore, the anti-humidity and anti-oxidation ability were also improved. DFS is one of the most efficient non-steroidal anti-inflammatory drug used in various diseases and is expected to develop new drag delivery method. Present intercalation seems to have a potentiality as a practical use in medical field.

\section{References}

1) G. Alberti, M. Casciola, and U. Constantino, J. Colloid Interface Chem., 107, 256 - 263 (1985).

2) E. González, L. Llavona, J. R. García, and J. Rodorígues, J. Chem. Soc. Dalton Trans., 1825-1829 (1989).

3) A. J. Khan, Y. Kanzaki, and Abe, M., J. Chem. Soc. Faraday Trans., 87, 2669-2674 (1991).

4) Y. Kanzaki, Y. Shimoyama, M. Tsukamoto, M. Okano, N. Suzuki, Y. Inoue, T. Tanaka, K. Koizumi, and Y. Watanabe, Chem. Pharm. Bull., 46, 1663-1666 (1998).

5) N. Suzuki, Y. Nakamura, Y. Watanabe, and Y. Kanzaki, Chem. Pharm. Bull., 49, 964-968 (2001). 